\title{
Caracterización y Evaluación de un Depósito de Caolín de "Los Azufres" Michoacán, México, para su Uso Industrial
}

\author{
J. GUILLEN A. S. BRIBIESCA V. Y R. ESCUDERO G.
}

Instituto de Investigaciones Metalúrgicas, Departamento de Cerámica,

Universidad Michoacana de San Nicolás de Hidalgo; Morelia Michoacán, México

\begin{abstract}
El caolín del depósito de "Los Azufres" del municipio de Zinapécuaro, estado de Michoacán, México, fue caracterizado y evaluado para su posible utilización industrial. Se estudió la fracción menor a $45 \mu \mathrm{m}$., por fluorescencia de rayos (FRX), difracción de rayos (DRX) y espectroscopía infrarroja (IR). Mostrando un alto contenido en sílice (70\%), asociada a las fases presentes en el mineral; cristobalita, tridimita y cuarzo seguidos de la caolinita con pequeñas cantidades de alunita. La distribución, morfología y el área superficial de las partículas fueron obtenidas por difracción de rayo láser, microscopía electrónica de barrido $(\mathrm{MEB})$ y por la técnica BET. Se realizó una caracterización térmica mediante análisis: térmico diferencial (ATD), térmico gravimétrico (ATG) y dilatométrico; revelando una fuerte expansión térmica del mineral desde temperatura ambiente hasta los $300^{\circ} \mathrm{C}$, seguido de una abrupta contracción entre $580^{\circ} \mathrm{C}$ y hasta $920^{\circ}$, relacionados con las transformaciones de fase de los polimorfos de la sílice, la deshidroxilación tanto de la alunita como de la caolinita y la desulfatación de la alunita. Las propiedades físicas tales como el color del mineral, grado de blancura, plasticidad, contracción lineal y densidad también fueron determinadas. Indicando que este mineral es recomendable para la industria del cemento y del plástico.
\end{abstract}

Palabras clave: caolín, recursos minerales, caracterización, mineral industrial.

Characterization and Evaluation of a kaolin deposit located in "Los Azufres" Michoacan, Mexico, for Industrial Use

In this work a kaolin ore from the region of "Los Azufres", state of Michoacan, Mexico, was characterized and evaluated in order to assess its use in the industry. A powder fraction less than $45 \mu \mathrm{m}$ were studied by X-ray fluorescence (XRF), x-ray diffraction (XRD) and infrared spectroscopy (IR). The results showed high silica content of $\sim 70 \%$, associated with minerals such as cristobalite, tridymite and quartz followed by kaolinite and a small amount of alunite. The distribution, morphology and surface area of the particles were obtained by Laser Diffraction, Scanning Electron Microscopy (SEM) and Brunauer Emmett Teller technique (BET). Thermal characterization was performed by differential thermal analysis (DTA), thermal gravimetric analysis (TGA) and dilatometric analysis. The thermal characterization results displayed a strong mineral thermal expansion from room temperature to $300^{\circ} \mathrm{C}$, followed by an abrupt contraction a temperatures between $580{ }^{\circ} \mathrm{C}$ to $920^{\circ}$. This could be associated with phase transformations of the polymorphs of silica, the dehydroxylation of the alunite and kaolinite and the desulfatation of alunite. The physical properties such as mineral color, degree of whiteness, plasticity, linear shrinkage and density were also determined. Finally whit the results obtained in this work, it can be concluded that the investigated ore is highly recommended for the cement industry and plastic.

Keywords: kaolin, characterization, mineral resources, industrial mineral.

\section{INTRODUCCION}

El caolín es un mineral que se produce por la descomposición de rocas feldespáticas, cuyo componente principal es la caolinita $\left(\mathrm{Al}_{2} \mathrm{O}_{3} \cdot 2 \mathrm{SiO}_{2} \cdot 2 \mathrm{H}_{2} \mathrm{O}\right)$. Es utilizado en diversas aplicaciones que van desde la industria cerámica, del cemento hasta otras industrias como la del papel, la pintura, en pesticidas, farmacéuticos, cosméticos etc., (1-4). México cuenta con importantes yacimientos de caolín generalmente de origen hidrotermal, siendo los Estados de Hidalgo, Zacatecas, Veracruz, Guanajuato y Michoacán, las entidades que han figurado entre los primeros lugares en la producción de este mineral en los últimos años (5). De manera general están compuestos por impurezas como el cuarzo, tridimita, cristobalita, halloysita y alunita (6). En el estado de Hidalgo se encuentra el mayor potencial geológico, el cual se extiende hasta el estado de Veracruz, principalmente en el municipio de Huayacocotla (6). En los yacimientos de caolín del estado de San Luis Potosí y Guanajuato, se ha encontrado adicionalmente la presencia de dolomita, muscovita, clorita y feldespatos como impurezas. Todos ellos presentan diversas variaciones en los contenidos de óxidos de hierro y titanio. Por lo que su valor económico es muy bajo debido a la gran cantidad de impurezas que contienen. $\mathrm{Su}$ procesamiento consiste únicamente en la extracción y molienda; en donde su clasificación se realiza de acuerdo a su coloración, comercializándose a granel por lo que éstos últimos son aplicados en la fabricación de cerámica artesanal. Por otra parte, se ha demostrado que las diversas aplicaciones de los caolines en procesos industriales, dependen de su mineralogía 
y las propiedades físicas de los mismos, así por ejemplo. El tamaño de partícula afecta la fluidez de pastas utilizadas en la industria cerámica, la plasticidad, la abrasividad y la facilidad de dispersión de las mismas. Las impurezas mineralógicas de los caolines afectan la refractariedad, resistencia mecánica, estabilidad térmica, resistencia a la humedad, la sensibilidad al secado y las transformaciones de fase que ocurren durante el quemado. El componente más importante es la alúmina $\left(\mathrm{Al}_{2} \mathrm{O}_{3}\right)$ el cual determina la refractariedad y resistencia mecánica de los materiales cerámicos, aumentando estas propiedades con el contenido de $\mathrm{Al}_{2} \mathrm{O}_{3}$. Por otra parte la plasticidad de la pasta cerámica y el índice de contracción, disminuyen significativamente con el aumento en el contenido $\mathrm{deSiO}_{2}(7)$. Finalmente el control en los contenidos de óxido de hierro y titanio afecta de manera significativa la producción de cerámica blanca, estos aportan cambios en la coloración y manchas durante el quemado de las piezas.

Este estudio permitirá evaluar las propiedades mineralógicas, térmicas y físicas que permitan proponer un destino más adecuado de este mineral a alguna de las diversas industrias reportadas, otorgándole un valor económico adicional a su explotación actual.

\section{MATERIALES Y METODOS}

El depósito, sujeto de este estudio se ubica en la sierra del mismo nombre, en el estado de Michoacán, México en el municipio de Zinapécuaro (ver Figura 1), que forma parte del Cinturón Volcánico Transmexicano atravesando al país de este a oeste (8). Se considera como hidrotermal de temperaturas moderadas (entre $200^{\circ}-300^{\circ} \mathrm{C}$ ), originado a causa de la alteración de las riolitas por corrientes geotermales que fluyen por diversas fracturas de las mismas. Ha sido sujeto de numerosos estudios geológicos, geofísicos y geotérmicos principalmente ya que en él se encuentra en las proximidades de una planta de energía geotérmica.

Para este estudio se colectaron muestras de diferentes zonas del depósito de caolín con diferentes contenidos del mineral arcilloso (caolinita). En una primera etapa se preparó una muestra representativa mediante homogenización y cuarteo, la cual fue sometida en primer lugar a una separación basta de los gruesos seguida por un proceso de molienda durante 4 horas, en un molino de acrílico con bolas de alúmina. Posteriormente el mineral fue clasificado con diferentes mallas en presencia de agua, realizándose un análisis mineralógico de cada fracción retenida por las diferentes mallas en un difractómetro (SIEMENS D5000), en el modo de análisis continuo empleando la radiación $\mathrm{K} \alpha$ de $\mathrm{Cu}$, con un incremento de $0.02^{\circ}$ y un tiempo de cuenta de $2 \mathrm{~s} /$ paso, además de un rango angular de 10 a $75^{\circ}$ en la posición $2 \theta$. Este análisis permitió seleccionar la fracción con mayor contenido de caolinita para llevar a cabo los estudios propuestos, la cual resultó ser la fracción menor a $45 \mu \mathrm{m}$ (tamiz 325). Seleccionada la muestra a estudiar se procedió con la siguiente etapa de caracterización y evaluación. Para completar el análisis mineralógico se obtuvo el espectro de infrarrojo (Bruker, modelo Tensor 27) en el intervalo de $500-4000 \mathrm{~cm}^{-1}$ en pastillas mezclándola con $\mathrm{KBr}$ prensadas de la muestra seleccionada. La composición química se llevó a cabo por espectrometría de fluorescencia de rayos $\mathrm{x}$ (Spectro Xepos III), con un tubo de rayos $x$ a 50 Watt con ventana de Pd. Para la cuantificación de todos los elementos químicos se utilizó el método Turbo Quant. La morfología de las partículas contenidas en la fracción con mayor contenido de caolinita fue examinada con un microscopio electrónico de barrido (MEB), (Jeol JSM-6400), equipado con un sistema de microanálisis (EDS), mientras que el área superficial fue obtenida por absorción de $\mathrm{N}_{2}$ por la técnica BET(Quanta sorb Jr). Por último
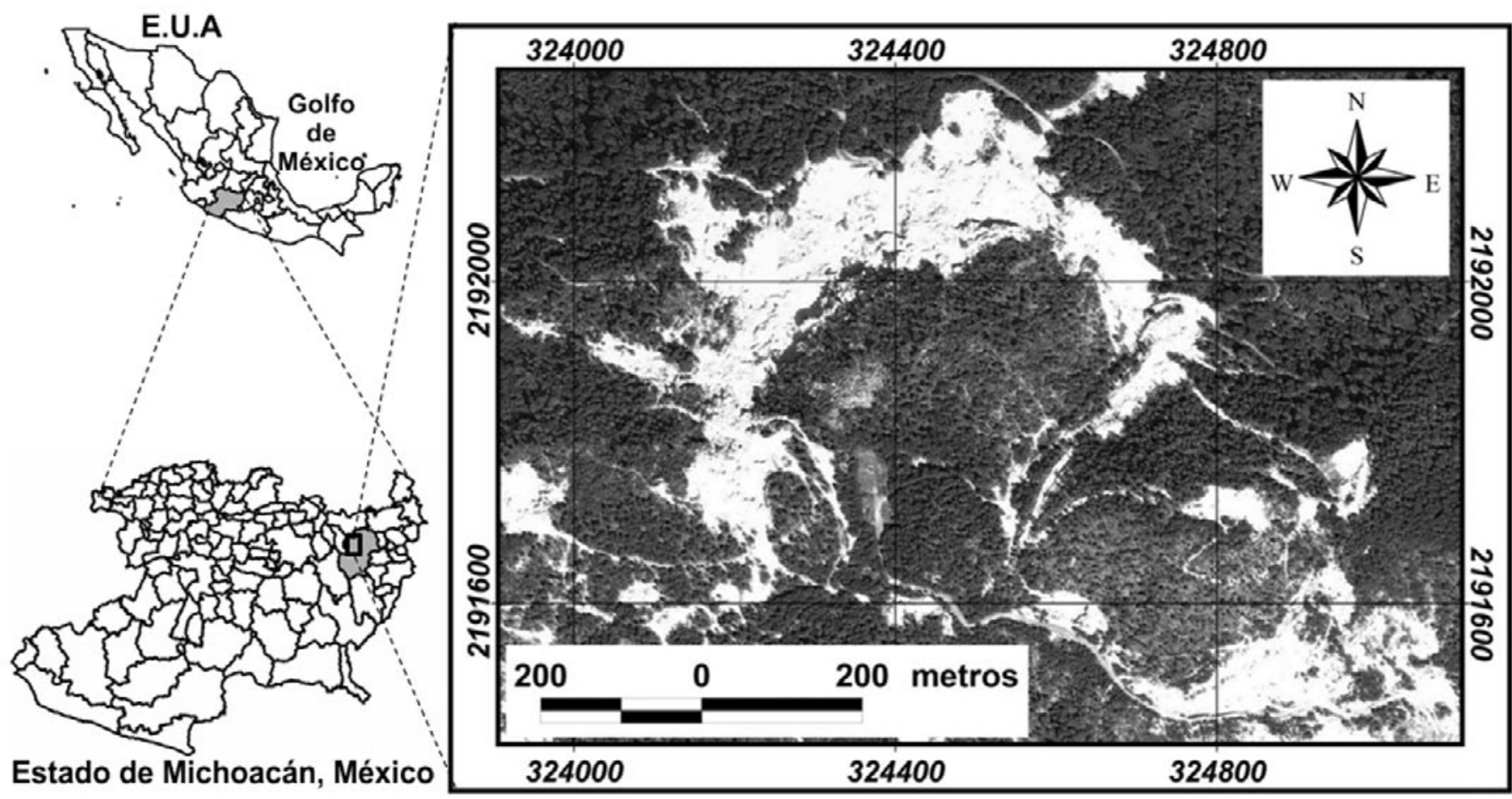

Figura 1. Localización geográfica del yacimiento “Los Azufres” en el Estado de Michoacán en México. 
se determinó el tamaño y distribución de tamaño de las partículas (DTP), (Beckman Coulter LS 1000).

Para la caracterización térmica se utilizaron tres diferentes tipos de análisis: un análisis térmico diferencial (ATD) para determinar la descomposición térmica de los minerales del caolín a una velocidad de calentamiento de $5^{\circ} \mathrm{C}$ por minuto bajo una atmósfera controlada con nitrógeno(TA Instruments, SDT Q600), equipado con un analizador térmico gravimétrico (ATG) que permitió determinar simultáneamente a la descomposición térmica, las pérdidas de peso asociadas a algunas transformaciones o reacciones químicas. Además se realizó un análisis dilatométrico para determinar los cambios dimensionales asociados a las transformaciones de los minerales presentes durante el calentamiento, para lo cual se prepararon probetas de $5 \mathrm{~mm} \times 5 \mathrm{~mm} \times 10 \mathrm{~mm}$ las cuales fueron llevadas desde temperatura ambiente hasta $1200{ }^{\circ} \mathrm{C}$ con una velocidad de calentamiento de $10^{\circ} \mathrm{C} / \mathrm{min}$. El equipo utilizado para este análisis fue un dilatómetro (Theta Dilatronic).

Finalmente se determinaron las propiedades físicas como son: El color del mineral, utilizando el sistema internacional CIE $L^{*} a^{*} b^{*}$, en un espectrofotómetro (X - Rite modelo CA22, con un software Match Rite). El grado de blancura se determinó (Brightmeter Micro S-5) y el índice de plasticidad se determinó por el método de Atterberg con ayuda de la copa de Casagrande. Además se evaluó el por ciento de contracción lineal. La densidad real se obtuvo en un picnómetro de desplazamiento (Micromeritics AccuPyc 1330).

\section{RESULTADOS Y DISCUSIÓN}

\subsection{Difracción de Rayos X (DRX)}

En el difractograma de rayos $\mathrm{x}$ del mineral seleccionado para esteestudio (fracción $<45 \mu \mathrm{m}$ ), que se muestra en la Figura 2 se indican los picos correspondientes a cinco fases cristalinas identificadas en esta fracción del mineral: cristobalita $\left(\mathrm{SiO}_{2}\right)$, caolinita $\left[\mathrm{Al}_{2} \mathrm{Si}_{2} \mathrm{O}_{5}(\mathrm{OH})_{4}\right]$, y en menor proporción cuarzo

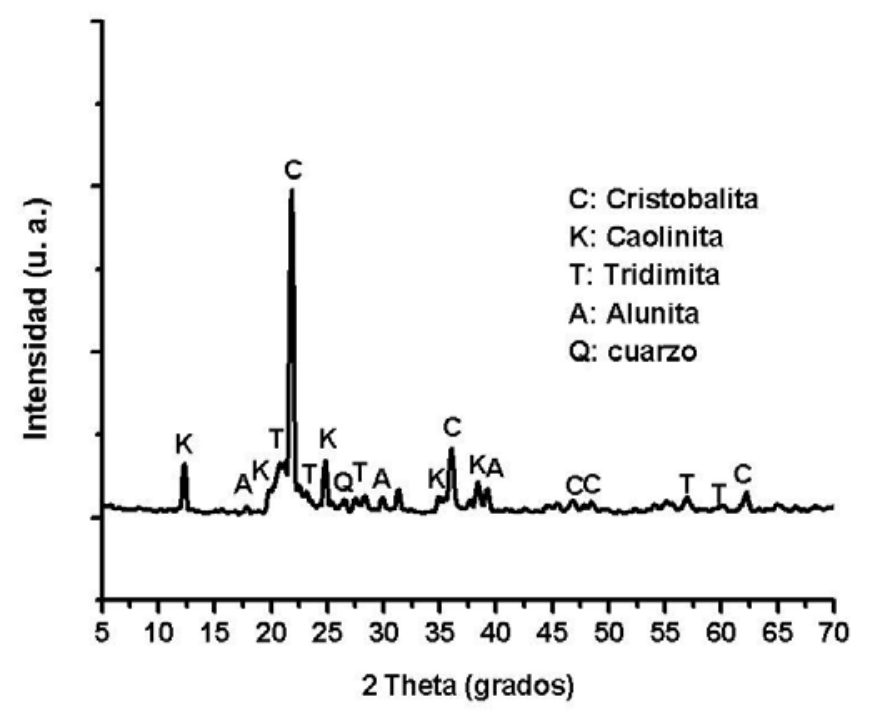

Figura 2. Difractograma del caolín de "Los Azufres", mostrando las fases cristalinas presentes.
$\left(\mathrm{SiO}_{2}\right)$, tridimita $\left(\mathrm{SiO}_{2}\right)$ y alunita $\left[\mathrm{KAl}_{3}\left(\mathrm{SO}_{4}\right)_{2}(\mathrm{OH})_{6}\right]$. En este difractograma se puede asociar el pico característico de la cristobalita (C) a $22,2^{\circ}$, el de la caolinita $(\mathrm{K})$ a $12,3^{\circ}$, el pico a $26,6^{\circ}$ al cuarzo (Q) y el pico alrededor de $21,7^{\circ}$ es atribuido a la fase tridimita $(\mathrm{T})$ mientras que a $30^{\circ}$ se presenta el de la alunita (A).

\subsection{Análisis por infrarrojo}

En la figura 3 se muestran los resultados de los estudios por IR del mineral. Los modos de vibraciones de estiramiento (Si-O) que se observaron en 790,9 $\mathrm{cm}^{-1}, 693,4 \mathrm{~cm}^{-1}, 538,8 \mathrm{~cm}^{-1}$ y $468,9 \mathrm{~cm}^{-1}$ son característicos de la presencia del cuarzo. Por otro lado, el pico en la posición $791 \mathrm{~cm}^{-1}$ se asigna a la vibración (Si-O) y es característica de la sílice en cualquier otra forma alotrópica (cristobalita y tridimita) (10). La aparición de bandas $\mathrm{n}(\mathrm{Si}-\mathrm{O}-\mathrm{Si})$ y $\mathrm{d}(\mathrm{Si}-\mathrm{O})$ también indican la presencia del cuarzo. Por otro lado, una amplia banda en $3450,4 \mathrm{~cm}^{-1}$ y $1633,4 \mathrm{~cm}^{-1}$ del espectro sugiere la posibilidad de agua adsorbida hidratando al mineral (11). La mayoría de las bandas encontradas a $3696,7 \mathrm{~cm}^{-1}, 3622,5 \mathrm{~cm}^{-1}, 3450 \mathrm{~cm}^{-1}, 1033,3$ $\mathrm{cm}^{-1}, 914,5 \mathrm{~cm}^{-1}, 790,9 \mathrm{~cm}^{-1}, 693,4 \mathrm{~cm}^{-1}, 538,8 \mathrm{~cm}^{-1}, 468,9 \mathrm{~cm}^{-1}$, muestran la presencia de caolinita (12). La vibración a 3489 $\mathrm{cm}^{-1}$, corresponde al modo característico de estiramiento de las unidades $\mathrm{OH}$ de la alunita (13).

\subsection{Análisis por Fluorescencia de Rayos X}

Los análisis químicos obtenidos por FRX se muestran en la tabla I, en la que se puede observar que el mineral estudiado muestra un alto contenido de sílice, $75 \%$ en peso y bajo contenido de $\mathrm{Al}_{2} \mathrm{O}_{3^{\prime}} 21 \%$; concordando estos resultados con el análisis mineralógico o sea que la sílice en sus diferentes formas es el principal componente del mineral. La presencia de $1.04 \%$ de $\mathrm{SO}_{3}$ a su vez, suponiendo que todo el azufre está contenido en la alunita nos indica que este mineral está presente en un 2.3 $\%$ en peso aprox. El contenido en óxido de hierro y óxido de titanio está por debajo del 1\%. Para calificar que tan adecuado puede ser un caolín para un sector en particular del mercado, 
el contenido de $\mathrm{Fe}_{2} \mathrm{O}_{3}$ y $\mathrm{TiO}_{2}$ es extremadamente importante (no más de 1\%) (14); ya que éstos óxidos colorean las piezas en la industria cerámica con el quemado, así por ejemplo, la porcelana o loza puede adquirir un color rojizo, marrón, amarillo, y púrpura, y particularmente altos contenidos de $\mathrm{TiO}_{2}$ disminuyen la translucidez de las porcelanas. Los óxidos de manganeso proporcionan el color naranja y el color negro. Los productos cerámicos también adquieren diferentes coloraciones en función de la relación de los diferentes óxidos presentes en las arcillas, por ejemplo, se obtiene el color amarillo, marrón claro y oscuro cuando se da la relación $\mathrm{Fe}_{2} \mathrm{O}_{3}$ : $\mathrm{CaO}=0,6-0,8$ y da color amarillo brillante y amarillo, si $\mathrm{Fe}_{2} \mathrm{O}_{3}$ : $\mathrm{CaO}=0,5-0,6$. Además, si el caolín contiene hierro en estado libre, pueden aparecer puntos oscuros en la superficie de los artículos. Estudios realizados han demostrado que el aumento del contenido de óxido de hierro y titanio (incluso por encima del $0,1 \%$ ), reduce la blancura en aproximadamente un $3 \%$ (5). El óxido de calcio, con frecuencia encontrado en caolines, actúa como un fundente. El efecto negativo de la adición de este óxido provoca daños a la estructura y a las propiedades mecánicas de los productos terminados (15).

En la tabla I además del análisis químico del mineral de caolín se muestra la composición química teórica de una caolinita (CT), el análisis químico del caolín de Georgia (CG) (uno de los depósitos de caolín más grandes de EUA), el de Villa de Reyes del estado de San Luis Potosí, México (VR) (16), el de dos yacimientos de arcilla del estado de Guanajuato, México (CG1 y CG2) (17) y de un caolín español (18). El contenido de $\mathrm{SiO}_{2}$ de los caolines mexicanos; el CA, el VR y el CG1 son muy altos si se comparan con los demás, por lo que estos "caolines" podrían ser mejor clasificados como arenas caoliníferas. Estas arenas están constituidas en la mayoría de los casos por un $80 \%$ de cuarzo y un $20 \%$ de minerales del grupo de la caolinita (18).

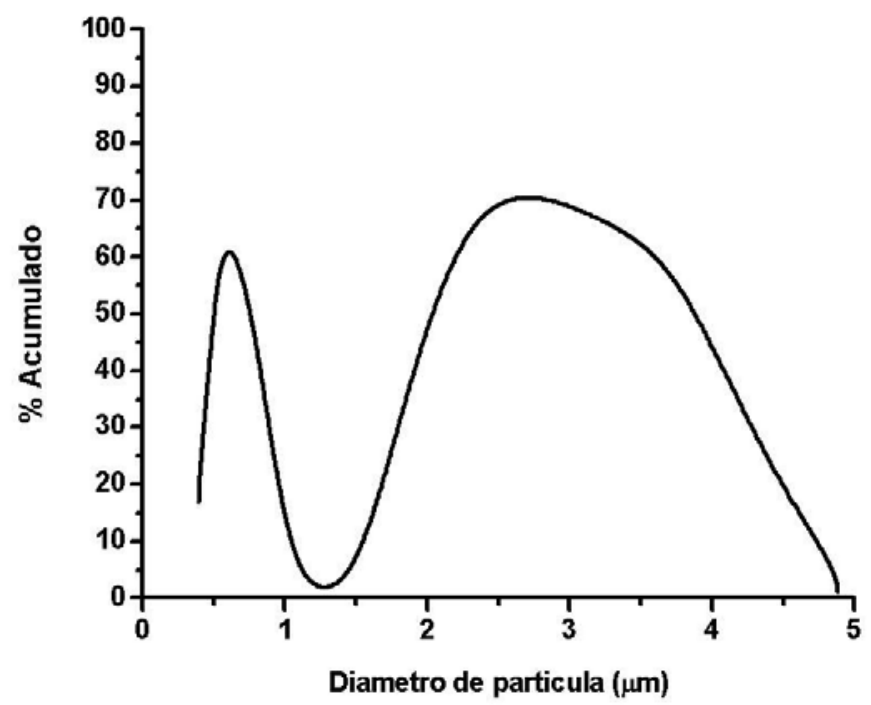

Figura 4. Distribución de tamaño de partícula del caolín $<$ malla 325

\subsection{Distribución de tamaño de partícula}

La DTP es un factor clave para el uso industrial de la caolinita. Por ejemplo las arcillas gruesas difieren de las arcillas finas en varias propiedades físicas incluyendo las ópticas (19). La DTP controla el brillo, la blancura, el encogimiento y la resistencia de la cerámica; y las propiedades mecánicas, ópticas y las características de impresión cuando se usa como relleno y como pigmento en el recubrimiento para la manufactura de papel.

Tabla I. Composición química del mineral de los Azufres Michoacán, Mexico (CA), del de Villa de Reyes del estado de San luis Potosí,

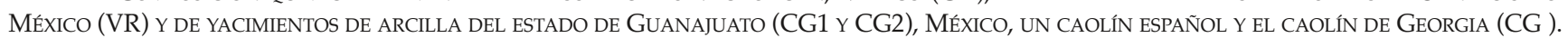

\begin{tabular}{|c|c|c|c|c|c|c|c|}
\hline \multirow{2}{*}{ Componente } & \multicolumn{7}{|c|}{ Caolines de diferentes regiones } \\
\hline & CA & VR & CG1 & CG2 & $\mathrm{CE}$ & CG & CT \\
\hline $\mathrm{SiO}_{2}$ & 75 & 72.63 & 78.2 & 50.6 & 51 & 45.30 & 46.3 \\
\hline $\mathrm{Al}_{2} \mathrm{O}_{3}$ & 21.07 & 15.04 & 13.7 & 30.9 & 36 & 38.38 & 39.8 \\
\hline $\mathrm{Fe}_{2} \mathrm{O}_{3}$ & 0.69 & 1.49 & 1.7 & 4.5 & 0.72 & 0.30 & ---- \\
\hline $\mathrm{CaO}$ & 0.01 & 0.7 & 0.1 & 0.5 & 0.02 & 0.05 & --- \\
\hline $\mathrm{MgO}$ & 0.03 & 0.55 & 0.8 & 0.5 & 0.05 & 0.25 & --- \\
\hline $\mathrm{K}_{2} \mathrm{O}$ & 0.53 & 0.65 & 2.9 & 1.0 & 1.3 & 0.44 & --- \\
\hline $\mathrm{TiO}_{2}$ & 0.32 & 0.34 & --- & ---- & 0.02 & 1.44 & ---- \\
\hline $\mathrm{Na}_{2} \mathrm{O}$ & 1.32 & --- & 0.2 & 0.1 & 0.03 & 0.27 & ---- \\
\hline $\mathrm{P}_{2} \mathrm{O}_{5}$ & 0.07 & --- & ---- & --- & ---- & ---- & ---- \\
\hline $\mathrm{SO}_{3}$ & 1.0142 & ---- & ---- & --- & ---- & ---- & ---- \\
\hline $\mathrm{PPC}\left(950^{\circ} \mathrm{C}\right)$ & ------ & 8.25 & 2.4 & 11.8 & 11.3 & 13.97 & 13.9 \\
\hline Total & 99.9 & & & & & & \\
\hline
\end{tabular}

${ }^{*} P P C$ se refiere a las perdidas por calcinación a $950^{\circ} \mathrm{C}$. 
La figura 4 muestra la DTP de la fracción que pasó por la malla 325, que corresponde al $57 \%$ del mineral inicial lavado. La curva bimodal (figura 4), muestra que el valor de la mediana es de aproximadamente $1.2 \mu \mathrm{m}$., y la moda corresponde a un valor de $2.7 \mu \mathrm{m}$. Lo anterior puede ser verificado con las imágenes obtenidas por $\mathrm{MEB}$, donde se observa que los tamaños de la caolinita son menores de $2 \mu \mathrm{m}$ y los de la cristobalita son mayores a $2 \mu \mathrm{m}$.
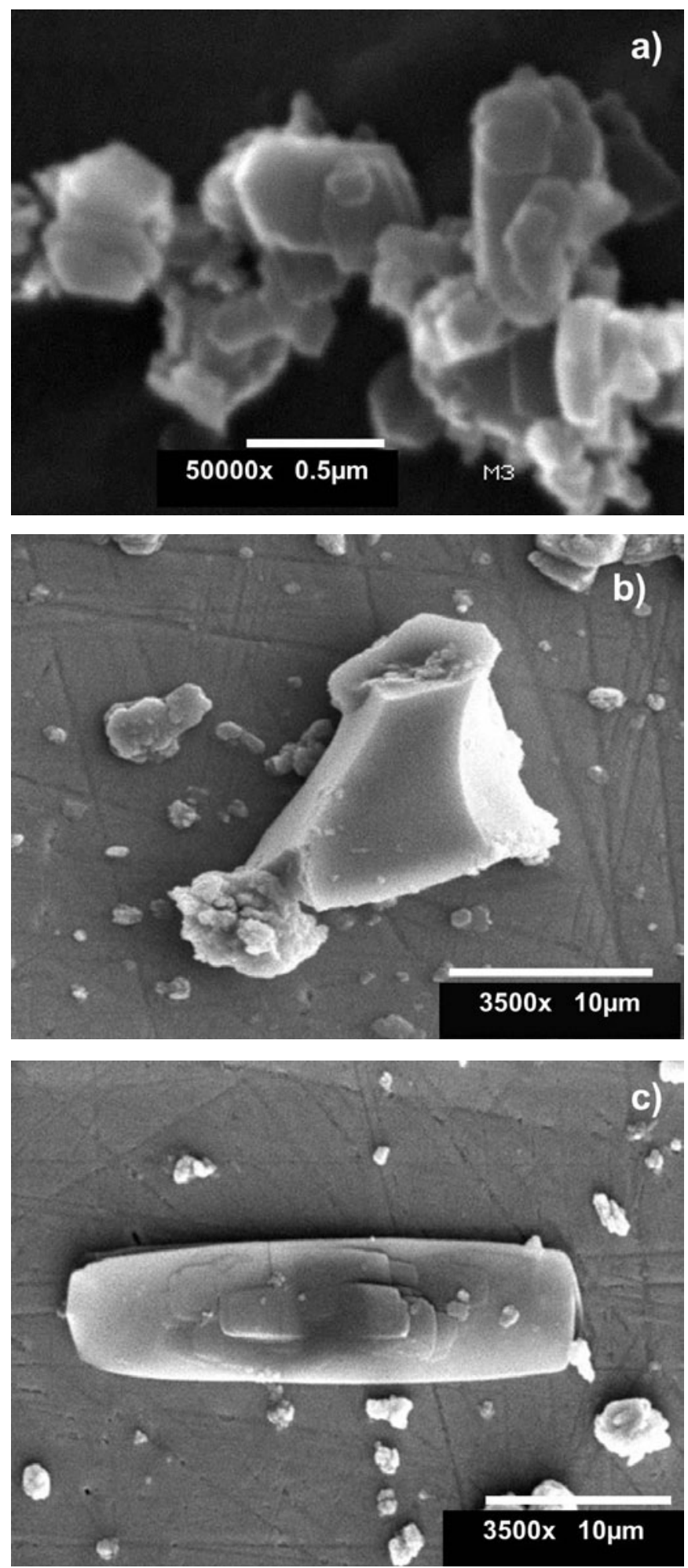

Figura 5. Micrografías electrónicas de las especies minerales presentes en el mineral de estudio.

\subsection{Microscopía Electrónica de Barrido (MEB)}

La Figura 5 muestra la morfología de algunas de las partículas contenidas en el mineral. La Figura 5a, corresponde a un agregado de partículas de caolinita, constituido por cristales subeudrales tanto en forma de placas hexagonales como seudohexagonales de diferentes tamaños menores a 2 $\mu \mathrm{m}$; resultados acordes a los obtenidos por la DTP. La Figura $5 b$, muestra cristales granulares los cuales corresponden al hábito cristalino de las partículas de cristobalita con tamaños menores a $30 \mu \mathrm{m}$ y la Figura $5 \mathrm{c}$, corresponde a cristales tabulares de tridimita menores a $30 \mu \mathrm{m}$. Estos análisis revelan claramente las diferentes morfologías y tamaños de partícula de cada una de las principales fases que constituyen el mineral.

Se ha reportado que las partículas de caolinita presentan un hábito hexagonal en el que la perfección de las láminas o placas va a depender de la cristalinidad de las partículas. Las partículas de caolinita bien cristalizada son hexagonales con sus bordes y esquinas bien definidos; mientras que las menos cristalinas presentan hexágonos con sus esquinas y bordes redondeados (20). Los minerales arcillosos del tipo de la caolinita se caracterizan por presentar áreas superficiales moderadas y tamaños de partícula que varía entre 0.5 y 10 micras (21).

\subsection{Análisis Térmico}

El análisis por dilatometría cuyo resultado se muestra en la Figura 6 presenta eventos importantes de expansión y contracción térmica en el mineral, asociados al cambio energético ocurrido durante el calentamiento de la muestra. Ocurre una ligera expansión térmica desde temperatura ambiente hasta $120^{\circ} \mathrm{C}$, y de ahí hasta $270^{\circ} \mathrm{C}$ se aprecia un cambio de pendiente, el cual es atribuido a la transición de la forma $\alpha$ a $\beta$ de la tridimita (16). Otros eventos importantes ocurren entre 270 y $360^{\circ} \mathrm{C}$ destacando una fuerte expansión térmica debido a la transición de la cristobalita $\alpha$ a $\beta$; otros

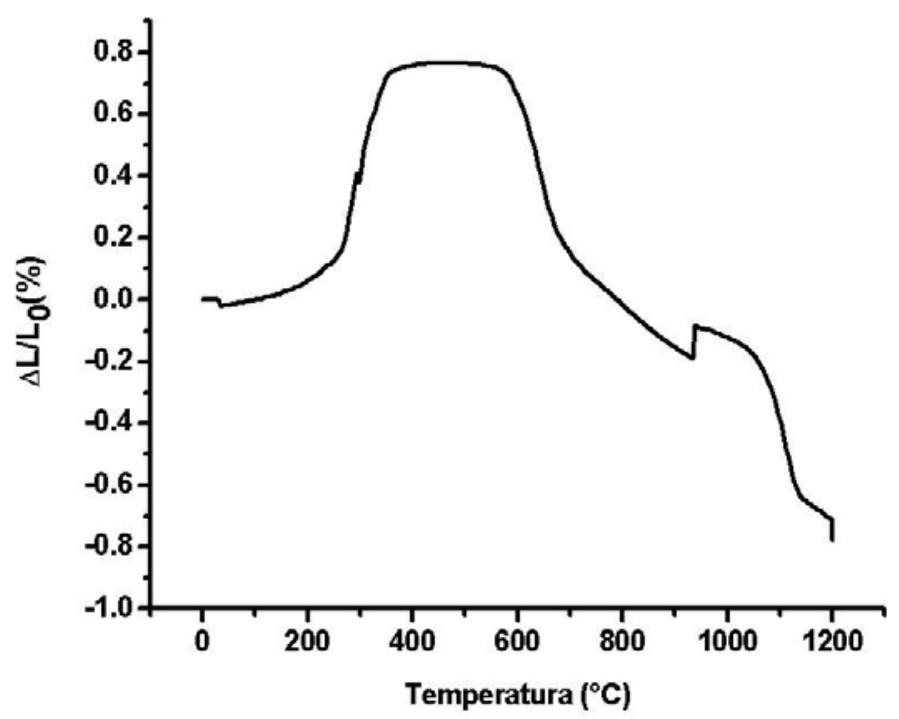

Figura 6. Análisis dilatométrico del caolín 


\begin{tabular}{|c|c|c|c|c|c|c|}
\hline Muestra & $\mathbf{L}^{*}$ & $\mathbf{a}^{*}$ & $\mathbf{b}^{*}$ & Reflectancia (\%) & Blancura (\%) & Área superficial $\mathbf{m}^{2} / \mathbf{g r}$ \\
\hline Caolín $<44 \mu \mathrm{m}$ & 92.45 & 0.32 & 4.25 & 70.46 & 79.4 & 12 \\
\hline Caolín Español & 81.73 & 2.26 & 11.36 & ------ & ----- & --- \\
\hline Caolín de SLP & 84.80 & 0.24 & 3.70 & ------ & ----- & - \\
\hline
\end{tabular}

autores han reportado que dicha expansión ocurre entre $200^{\circ}$ y $300^{\circ} \mathrm{C}(22)$. Entre $360^{\circ}$ y $560^{\circ} \mathrm{C}$ se observa un rango de estabilidad, seguida de una contracción abrupta hasta $680^{\circ} \mathrm{C}$ atribuida a la transición del cuarzo de su forma a a b y la pérdida de agua estructural de la caolinita además de la desulfatación de la alunita. En el rango de $680^{\circ}$ a $932^{\circ} \mathrm{C}$, ocurre otro cambio de pendiente que puede ser asociado a la transformación de la tridimita a cuarzo (23). El pico observado a $980^{\circ} \mathrm{C}$, corresponde a la formación de espinela. Y finalmente se observa una última contracción atribuida al inicio de la sinterización de las partículas o la formación de nuevas fases (24).

Las curvas del ATD y del ATG se muestran en la Figura 7 ambas están relacionadas con cambios físicos y químicos de la muestra. En la curva ATD se observan varios picos pequeños a $63^{\circ}, 88^{\circ}, 109^{\circ}, 160^{\circ}, 198^{\circ}, 270^{\circ}, 368^{\circ}, 509^{\circ}, 572^{\circ}, 749^{\circ} \mathrm{C}$ y $980^{\circ} \mathrm{C}$. El primero corresponde a la remoción de humedad adsorbida físicamente, el segundo, tercero y cuarto corresponden a las transformaciones a baja temperatura de la tridimita, el quinto y sexto a la transformación de $\alpha$ a $\beta$ de la cristobalita (20). Cabe mencionar que el mineral contiene pequeñas cantidades de alunita, ocurriendo el comienzo de la deshidroxilación de ésta, se encuentra por encima de $220^{\circ} \mathrm{C}$; sin embargo, el pequeño cambio de pendiente que puede ser asociado a este fenómeno en este caso ocurre a $368^{\circ} \mathrm{C}$. El octavo corresponde la formación de metacaolín. El pequeño pico observado a $572^{\circ} \mathrm{C}$ corresponde a la transformación de cuarzo de $\alpha$ a $\beta$. Finalmente el último corresponde a la formación de la

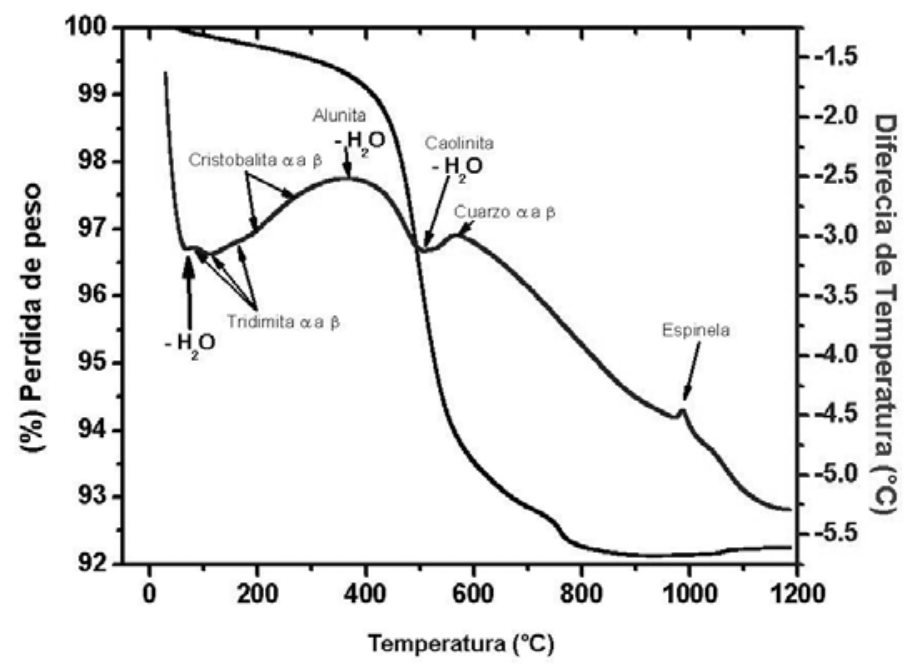

Figura 7. Análisis térmico diferencial y gravimétrico del caolín espinela. De la curva ATG se estimó una pérdida en peso total del $9 \%$ debido a estos procesos de deshidroxilación incluida la desulfatación de la alunita que aunque en la curva del ATD no se muestra el pico en la curva ATG se observa un pequeño cambio de pendiente alrededor de $730^{\circ} \mathrm{C}$ temperatura reportada para este proceso (13). Mediante el análisis de la curva de ATG y el análisis químico realizado al mineral fue posible estimar el porcentaje de caolinita en el caolín, tomando en cuenta la cantidad de alunita calculada con anterioridad. La pérdida en masa debida a la deshidroxilación de la arcilla fue de aproximadamente $6 \%$ y como la pérdida en masa de un caolín de composición ideal es de $14 \%$ (25, 26), por lo tanto podemos estimar que el mineral contiene aproximadamente un $40 \%$ de caolinita.

Los estudios realizados demuestran que la presencia de cristobalita y tridimita, puede ocasionar dos eventos importantes de contracción térmica durante el enfriamiento, el primero ocurre desde $360^{\circ}$ hasta $260^{\circ} \mathrm{C}$, y el segundo de ésta última temperatura hasta temperatura ambiente, por lo tanto, si este mineral se destina a la fabricación de piezas cerámicas puede ocurrir la ruptura de las piezas. En la industria del cemento, se ha reportado que la presencia de las fases de la sílice unidas al vidrio, favorecen el abrasamiento del clínker, especialmente del clínker blanco (16). Otros estudios, han demostrado que bajos contenidos de caolinita (48\%) en un caolín permiten producir un metacaolín con excelentes propiedades puzolánicas a edades largas y con efectos importantes y positivos en las propiedades de durabilidad de las mezclas adicionadas $(12,27)$. En la industria de los materiales refractarios, cuando predominan las fases cristalinas del $\mathrm{SiO}_{2}$ : cuarzo, tridimita y cristobalita, las propiedades mecánicas disminuyen; debido a las transformaciones de fase que ocurren a relativamente bajas temperaturas que suelen ir acompañadas por grandes cambio en el volumen (28). Por lo anterior y además por el bajo contenido de álcalis en el mineral de estudio, éste mineral puede ser adecuado para su uso en la fabricación de cemento bajo en álcalis y factible para su empleo en la elaboración de cemento blanco.

\subsection{Propiedades físicas}

Las propiedades físicas del caolín dependen de la mineralogía ya que cada componente mineral contribuye de manera positiva o negativa a modificarlas. Estas propiedades también dependen del tamaño y distribución de partícula, de su forma, su cristalinidad, área superficial y el grado de pureza principalmente (4). En la tabla II, se muestran los valores determinados del color en el sistema CIE del mineral de estudio y para comparación se muestran los valores de un caolín comercial español y otro beneficiado; L* representa la 
TAbla III. LÍMites DE ATTERberG DEL MiNERAl DE ESTUdio.

\begin{tabular}{|c|c|c|c|c|c|}
\hline Mineral & LP (\%) & LL (\%) & Ip (\%) & C.L (\%) & Densidad \\
\hline Caolin $>45 \mu \mathrm{m}$ & 34.17 & 44.68 & 10.51 & 3.1 & 2.5 \\
\hline
\end{tabular}

luminosidad (claro u oscuro); $\mathrm{a}^{*}$ indica la orientación al color rojo (positivo) o al verde (negativo) y b* la tendencia del color al amarillo (positivo) o al azul (negativo), lo anterior indica que el mineral presenta buena blancura comparada con los otros caolines (16) debido al bajo contenido de hierro y titanio presentes en el mineral.

Los límites de Atterberg: límite líquido (LL), límite plástico (LP) e índice de plasticidad (Ip) y la contracción lineal del mineral se presentan en la Tabla III. De acuerdo a los valores determinados de límite líquido y el índice de plasticidad, el mineral se puede clasificar como una arcilla inorgánica de baja plasticidad de acuerdo al diagrama de Casagrande (29).

La baja plasticidad en el mineral de caolín se atribuye a los altos contenidos de cristobalita en el mineral $(60 \%$ aproximadamente).

\section{CONCLUSIONES}

De la caracterización mineralógica, morfológica y física, del mineral de caolín de los Azufres, Michoacán, México, se derivan las siguientes conclusiones:

- El caolín está constituido principalmente por cristobalita y caolinita, acompañado de cantidades minoritarias de tridimita, alunita y cuarzo. La morfología se observa en forma clara para las especies minerales contenidas en el caolín: cristales granulares y tabulares que corresponden a las partículas de cristobalita y tridimita respectivamente, con un tamaño en la rango aproximado de 1 a $30 \mu \mathrm{m}$. Los cristales subhedrales en forma de placas hexagonales y pseudohexagonales de las partículas de la caolinita, se presentan a tamaños menores a $2 \mu \mathrm{m}$.

- Los estudios realizados al mineral de caolín (fracción menor a $45 \mu \mathrm{m}$ ), permiten proponer la aplicación de este mineral en la industria del cemento blanco y como relleno en la industria del plástico, debido a las fases alotrópicas de la sílice presentes en el mineral y los bajos contenidos de hierro, titanio y álcalis; además del grado de blancura del mineral, no requiriendo procesos adicionales de beneficio ya que cumple con las especificaciones para tal destino.

Por otro lado, si se desea elevar la calidad del mineral para destinarlo a alguna otra industria por ejemplo; la industria cerámica, es necesario establecer un proceso de concentración de la especie caolinita. Previamente estudiando los valores de propiedad de las principales especies contenidas en el mineral.

\section{BIBLIOGRAFÍA}

1. C. C. Harvey and. H. H. Murray, Industrial clays in the 21st century: A Perspective of Exploration, Pechnology and Utilization, Applied Clay Sci., 11 285-310 (1997).

2. H. H. Murray, Applied Clay Mineralogy Today and Tomorrow, Clay Miner, 34, 39-49 (1999).

3. H. H. Murray, Traditional and new applications for kaolins, smectite and palygorskite: A General Overview., Applied Clay Sci., 17 207-221 (2000).

4. H. H. Murray, Applied clay minerals: occurrences, processing and application of kaolins, bentonites, palygorskite-sepiolite, and common clays I. Bloomington: Indiana, U.S.A, (2007).

5. Servicio Geológico Mexicano, web.www.sgm.gob.mx.

6. F. Legorreta y col., Caracterización y separación gravimétrica de arenas de caolín procedente de agua blanca de Iturbide, Hidalgo (México), XIX International Conference on Extractive Metallury, Saltillo, Coahuila México. Mayo (2010)

7. V. V. Mikhalev. and A. S. Vlasov, Kaolins for production of sanitary ceramics, Glass and Ceramics, 63,9 293-297 (2006).

8. E. G. Partida, Evidencias de evolución de un fluido básico a ácido a apartir del analisis de la alteración hidrotermal del campo geotérmico de los Azufres, Michoacan, R. M. C. G.,17,1, 76-82 (2000).

9. V. H. G. Monroy, La caldera de los Azufres y su relación con el sistema regional E-W.," R. M. C. G., 4, 1, 49-61 (1989).

10. Y. M. Vargas y col., Caracterización espectroscópica, química y morfológica y propiedades superficiales de una montmorillonita Mexicana, R. M. C. G., 25(1) 135-144 (2008).

11. P. S. Nayak y B. K. Singh, Instrumental characterization of clay by XRF, XRD and FTIR, Bull. Mater. Sci., 30, 3, 235-238 (2007).

12. J. Torres y col., Análisis comparativo de caolines de diferentes fuentes para la producción de metacaolín, Rev. LatinAm. Metal. Mat., 31, 1 35-43 (2011)

13. R. L. Frost y D. L. Wain, "A Thermogravimetric and infrared emission spectroscopic study of alunite, Journal of Thermal Analysis and Calorimetry, 91, 1, 267-274 (2008)

14. G. S. Maslennikova, N. Solodkaya, M. Shamrikov, Use of kaolins from different deposits in the production of fine ceramics," Glass and Ceramics, 61, 7, 257-266 (2004).

15. M. T. Makhsudova, Casting properties of slips made from tankerissk clays containing a large amount of chlorides, Steklo Keram, 5, 20-22 (1977).

16. F. Vázquez, L.M. Torres, L. L. Garza, A. Martínez y W. López, Caracterización por XANES, análisis mineralógico y aplicación industrial de un depósito de caolín de méxico, Materiales de Construcción, 59 ,294, 113-121 (2009).

17. E. Ramos y col., Caracterización de arcillas del Estado de Guanajuato y su potencial aplicación en cerámica, Acta Universitaria, Universidad de Guanajuato, Guanajuato, México, 12, 1, 23-30 (2002).

18. L.A. Diaz Rodriguez, R. Torrecillas, Arcillas cerámicas: una revisión de sus distintos tipos, significados y aplicaciones, Bol. Soc. Esp. Cerám. Vidrio 41, 5, 459-470 (2002).

19. A. A. A.-S. y L. X. Rong, Characterization and evaluation of alga of kaolin deposits of yemen for industrial application, American J. of Engineering and Applied Sciences 2, 2 292-296 (2009).

20. W. D. Callister, Introducción a la ciencia e ingeniería de los materiales I. Barcelona, España, (2007).

21. S. A. Siradz, Mineralogy and chemistry of red soils of Indonesia, Tesis Doctoral, Faculty of Agriculture, The University of Western Australia, (2000).

22. J. B. Baldo, W. N. dos Santos, Phase transitions and their effects on the thermal diffusivity behaviour of some $\mathrm{SiO} 2$ polymorphs, Cerâmica, 48, 307, 172-176 (2002). 
23. Y. B. Chiang, D.P. Kingery, W.D., Physical ceramics: principles for ceramic science and engineering: J. Wiley, (1997).

24. G. P. Souza, R. Sanchez, J. N. F. de Holanda, Characteristics and physicalmechanical properties of fired kaolinitic materials, Cerâmica 48, 306, 102 - 107 (2002).

25. W. M. Carty and C. W. Sinton, Science of whitewares II :the origins of kaolinite-implications for utilization, The American Ceramics Society, Westerville, Ohio, 3-12 (2000).

26. W. M. C. a. C. W. Sinton, Science of whitewares II: mineralogy of ceramic grade kaolins from the southeastern united states, The American Ceramics Society, Westerville, Ohio, 77-88 (2000).

27. J. Torres, R. Mejia, Influencia de la composición mineralógica de los caolinines sobre el desempeño de morteros adicionados con MK, Rev. LatinAm. Metal. Mat., 74, 153, 61-67 (2007).

28. N. P. Cheremisinoff, Handbook of ceramics and composites: synthesis and properties 1. New York, (1990).

29. Pérez Ayala y col., Beneficio de una arcilla caolinítica de la región de Barichara (Santander) para la fabricación de refractarios, Dyna, 164 29-38 (2010)

Recibido: $11 / 11 / 2011$

Aceptado: $11 / 07 / 2012$ 